\title{
A Study on Food Safety Risk Analysis under the Condition of Traditional Agriculture Jingyuan Yin ${ }^{1}$ Haiyan Gao $^{2}$ Wei Pei ${ }^{2}$ \\ ${ }^{1}$ School of Computer Engineering and Science, Shanghai University Shanghai 200072, China, jyyin@staff.shu.edu.cn \\ ${ }^{2}$ School of Life Sciences, Shanghai University, Shanghai 200444, China
}

\begin{abstract}
With the development of economy, the problem of food safety has caused extensive concern. Agricultural production node is on the front of the food safety management chain. The combination of which and modern economic social development is an important character to reflect the food safety situation in China. In a way of food safety risk analysis, the major problem under the condition of traditional agriculture is the whole product and sale process does not match with the modern information-based management. For one reason or another, it is hard for people to acquire kinds of planting and breeding data, which bring the great difficult to the work of food safety risk evaluation and supervision. The premise to solve the problem is to invest more in agriculture, substantially improve the quality of persons engaged in agricultural production in our country, change some backward product process modes under the condition of traditional agriculture as soon as possible and quicken the pace of informatization construction in rural area. And enacting and improving relevant laws and regulations of food safety by borrowing experience from the developed countries.
\end{abstract}

Keywords: Traditional agriculture, food safety, risk analysis.

\section{传统农业条件下的食品安全风险分析研究 尹京苑 ${ }^{1}$ 高海燕 $^{2}$ 裴炜 $^{2}$ \\ 1.上海大学计算机工程与科学学院, 上海 200072 \\ 2. 上海大学生命学院, 上海 200444}

摘要: 随着经济的不断发展, 食品安全的问题会越来越引起人们的关注。作为食品安全管理链条的最前 端一农业生产环节，其与现代经济社会发展的融合度是体现我国食品安全状况好坏的一个重要特征指标。而从 食品安全风险分析的角度来看, 传统农业条件下进行此项工作的最大问题在于, 其整个生产销售过程与现代社 会的信息化管理格格不入, 由于种种原因, 人们很难真实有效地获取各类种植养殖数据, 这给食品安全的风险 评估与监管工作带来了巨大的困难。而解决这一困难的前题是加大农业投入, 切实提高我国农业生产人员的素 质，尽快改变传统农业条件下的一些落后生产加工模式，加速农村信息化建设步伐。同时借鉴国外发达国家的 先进经验, 建立完善与之相关的食品安全法律法规体系。

关键词：传统农业，食品安全，风险分析

\section{1. 引言}

随着工业化的进程和科技的进步, 国际食品安 全事件不断发生，1999 年比利时的 “二恶英事件” 事件爆发之后, 食品安全问题愈演愈烈, 成为世界 各国关注的焦点。如何保证食品的安全, 维护消费 者权利, 促进食品贸易健康发展, 是摆在各国面前 必须思考解决的问题。过去食品安全的管理是以对 不安全食品的立法、清除市场上的不安全食品和责 任部门的认可等项目的实施为基础的。然而, 这些
传统的管理方法由于缺乏预防性手段, 因此对食品 安全现行存在及可能出现的危害不能做出及时的应 答。

风险分析是近 20 年发展起来的一种解决食品安 全问题的手段, 可为食品安全决策提供系统和规范 的科学数据。随着食品问题的不断复杂和多样, 传 统的监管方式表现出了极大的效果和效率方面的无 能。而风险分析这种监管方式由于能够最有效地将 风险控制在萌芽状态, 因此, 起到了很好地预防食 品安全风险的作用 ${ }^{[1]}$ 。食品风险分析代表了现代科 
学技术最新成果在食品安全管理方面实际应用的发 展方向, 已经成为世界各国普遍遵循的规则, 是制 定食品安全标准和解决国际食品贸易争端的重要依 据。

农业是食品的基础, 而中国是一个农业历史悠 久的国家, 农业在整个国民经济中占有重要的地 位。食品安全问题的发生与农业生产条件有着很大 的关系, 我国目前的农业在很大程度上还是传统 的、原始的小农小本耕作经营, 高成本、低效率是 它的显著特征, 整个农业生产无序又疏于管理, 科 技含量和知识含量很低, 农业产业基础非常薄弱, 与发达国家相比, 农业的落后程度远远超过其他行 业。我国人口众多, 农业资源紧缺, 农业发展受到 很大的资源制约, 加上长期以来实行高度集中的计 划经济体制, 商品经济没有得到充分发展, 这些特 殊的国情, 决定了我国未来农业发展必须走一条适 合中国国情的、具有中国特色的、又能与国际接轨 的农村改革和发展道路。

\section{2. 食品安全风险分析的基本原理}

食品安全风险分析始于 20 世纪 80 年代末, 最先 应用于航天食品。1998 年在罗马召开的FAO/WHO 联合专家咨询会上, 形成了《风险情况交流在食品 标准和安全问题上的应用》的报告, 标志着食品安 全风险分析的理论框架已经形成。如图1所示 ${ }^{[2]}$, 食 品安全风险分析包括风险评估、风险管理和风险交 流这 3 个互为前提、相互作用的部分。这 3 个部分形 成了一个科学的管理框架, 其基本原理如下。

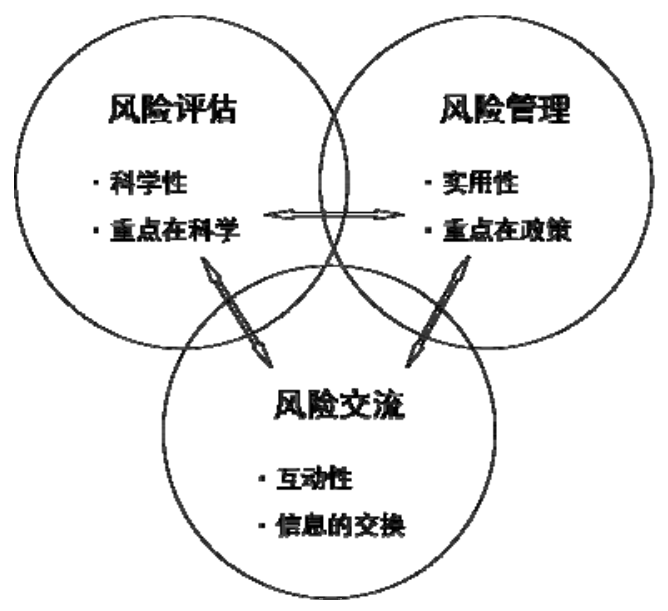

图 1 食品安全风险分析各部分之间的关系

风险分析是指对食品中可能存在的危害进行预 测, 并在此基础上采取规避或降低危害影响的措
施，包括风险评估、风险管理和风险交流三个部 分。

风险评估是以科学为基础对食品可能存在的危 害进行界定, 特征描述, 暴露评估和描述的过程。 具体说就是通过现有的资料包括毒理学数据、污染 物残留数据、统计手段、暴露量及相关参数的评估 等系统的、科学的步骤, 对食品中生物、化学或物 理因素对人体健康产生的不良后果进行识别、确认 和定量, 决定某种食品有害物质的风险。它为风险 管理提供依据, 其实施主体是食品科学、卫生学、 毒理学等方面的科学家。

风险管理是根据风险评估的结果，对公平贸易 的影响程度进行评估, 选择和实施适当的管理措 施, 尽可能有效地控制食品的风险, 从而保障大众 的健康。其目标是选择措施, 把食品风险降低到可 接受的水平, 风险管理的实施主体是政府, 政府主 要通过制定食品中有害物质或微生物的最高限量、 卫生标准、食品标签标准、食品法律法规, 以及采 取公众食品安全教育等措施, 保障公众健康。一般 包括风险评价、风险管理政策评估、执行管理决定 以及监控和审查几个过程。

风险交流是指在食品安全科学工作者、管理 者、生产者、消费者以及感兴趣的团体之间进行风 险评估结果、管理决策基础意见和见解传递及交换 的过程。这样通过公开、透明的信息交流，使各方 全面了解影响食品安全的各种危害、危害的特征、 危害的严重程度、危害的变化趋势、最高风险人 群、风险人群的特点和规模、风险人群对风险的接 受程度、风险人群的利益等信息。风险交流在于使 社会公众, 尤其是广大消费者参与到食品安全管理 中去, 促进政府综合考虑各种信息, 提高决策的透 明度和科学性, 制定更加合理的食品安全政策, 将 食源性风险减少到最低限度, 实现食品安全水平的 不断提高。风险交流的目标就是确保将所有关于有 效风险管理的信息和意见考虑进决策过程中 ${ }^{[3,4,5}$, ${ }^{6]}$ 。

\section{3. 传统农业条件下的食品安全问题}

“传统农业”与 “现代农业” 的划分, 是以农 业生产过程中生产技术手段应用的水平、程度和范 围来确定的。凡是大量应用现代先进的科学技术, 使劳动生产率大幅度提高, 并有较发达的社会经济 环境和其他高技术配套产业的密切配合, 为现代农 业; 如果继续使用常规的生产技术手段, 主要还是 人畜耕作、人工收种、靠天吃饭等, 则是传统农 业。目前, 中国农业虽然取得了许多可喜的成绩, 
但从总体上说, 特别是以占总人口百分之八十的农 民在广大农村所从事的生产来说, 占主导地位的还 是以手工劳动为主的耕作方式, 人力畜力使用还比 较普遍, 农业劳动生产率还比较低, 所通行的仍然 是以农户为单位的小规模生产, 因此仍属于传统农 业方式的范畴 ${ }^{[7]}$ 。

随着人类活动的发展, 食品行业日益国际化, 开放、合作等因素使食品供给变得异常复杂, 因此 现代食品安全问题作为一个社会及经济问题的存 在, 也随着食品经济体系的变化而变化, 并随其发 展而发展。不同的农业生产条件下, 食品安全产生 的原因与存在的问题也各有差异, 中国作为一个仍 处于传统农业方式下的国家。一般而言, 食品供应 链是由农业、食品加工业和物流配送业等相关企业 构成的食品生产与供应的网络系统, 主要围绕食品 生产、食品供应、食品物流与食品需求等四个主要 领域来组织实施的。处于建设初期的我国食品供应 链管理领域, 一方面承受着国际化竞争的巨大压 力, 一方面面临着许多亟待解决的问题 ${ }^{[8]}$ 。

\section{1 生产环节的食品安全问题}

主要是农产品源头污染问题。一方面, 随着社 会的不断发展, 人口的不断增加, 人们对粮食的需 求也在不断增加, 因此为了满足人们日益增长的对 食物的需求, 在现实中, 农民往往投入更多的农 药、化肥来达到这种目的。同时, 工业的发展让农 村环境受到污染, 工业三废、城市废弃物的大量排 放, 造成许多有毒、有害物质排放到土壤、水和空 气中。造成了农产品的种植、养殖正在不同程度地 受到农药、化肥、三废的影响与污染, 人体在食用 了这些被污染的农产品后不仅将产生直接的健康危 害, 而且还可造成食源性疾病的增加, 同时这些化 学性、生物性的污染, 往往会在食物链中进行蓄 积, 给食品安全的风险分析与评估带来挑战。

\section{2加工环节的食品安全问题}

我国农业生产目前的现状为, 在相当一部分地 区，农业还是小农经济的模式，农业设备极其落 后, 生产工艺粗粘落后, 管理混乱, 许多生产者忽 视了质量安全, 甚至根本没有质量安全的概念, 这 是加工环节食品安全问题产生的主要原因。

这些安全问题主要表现在: 一是企业生产规模 过小, 管理混乱, 未能按照工艺要求操作。以大米 为例, 占总销量 $50 \%$ 以上的产品都是由 10 人以下、 设施简陋的家庭作坊式企业生产, 这样厂家为追求 利润最大化, 往往减少了设备、设施和管理的投
入, 导致产品在加工、咜运过程中受到污染而出现 安全问题 ${ }^{[8]}$ 。

二是超量使用食品添加剂, 甚至非法使用非添 加物而引起食品安全问题。为了改善食品品质, 延 长保质期, 在食品加工中必须使用一定数量的食品 添加剂, 但一些生产企业却为了降低生产成本, 不 惜滥用非法使用一些添加物, 增加了市场监管的难 度。

三是应用新技术、新工艺、新原料带来的问 题。如转基因技术、现代生物技术、益生菌和酶制 剂等技术在食品中的应用, 辐照食品的安全性评价 等已经成为人们关注的热点问题。

\section{3流通环节的食品安全问题}

受经济发展等许多因素的影响, 许多农产品或 食品仍然以传统的批发市场和农贸市场流通的形式 进行, 这样存在着许多安全隐患。由于检测农药残 留量等会影响农产品的进场流量, 因此许多小批发 市场和农贸市场缺乏有效的管理手段和机制, 同时 由于销售场所简陃、卫生条件缺乏, 许多新鲜果 蔬、水产品等产品极易腐败变质, 增大了食品安全 问题发生的几率。

据统计，我国每年有总值 750 亿元人民币的食 品在运送过程中发生腐败。许多食品生产企业为了 追求利益的最大化, 对物流设备的保养检修的重视 与投资力度不够, 尤其是冷链物流及其相关设施严 重落后且缺乏维修更新, 远远达不到食品保藏的温 度要求, 致使大部分食品在运输过程中变质或质量 水平降低, 结果不但造成了经济损失, 而且影响了 企业的信誉和形象。

\section{4消费环节的食品安全问题}

现阶段, 随着现代家庭结构的缩小与人口流动 性的增大, 人们在外就餐消费等活动大大增多, 使 得消费环节的食品安全问题变得更加严重。一方 面, 一般消费者对日常使用的食品都会产生相关的 信息判断, 这种判断直接左右了消费者的购买与使 用行为，久而久之甚至养成一种消费爱好或习惯; 另一方面由于受消费者相关知识水平等的限制、生 活习惯的影响、食品安全信息的不对称的影响, 造 成这些消费习惯和信息判断上的不准确, 往往引领 消费者走向一个对于食品的误区，从而使人们很难 根据自身的力量来保护自己, 造成了消费环节二次 污染等许多食品安全问题频繁发生。

3.5 食品安全问题产生的原因 
3.5.1 经济利益的驱动是产生食品安全问题最直接的 原因

在社会主义市场经济条件下, 从事农产品种 植、食品生产、加工、销售的个人或企业都追求自 身的经济利益。为了追求经济利益的最大化, 大量 以次充好, 以假乱真的食品安全事件频繁发生。致 使在生产环节超量使用农药、化肥, 滥用激素、抗 生素; 在加工环节使用劣质原料或滥用及违法使用 一些添加物; 在销售环节销售一些假冒伪劣产品。 3.5.2 法律法规体系的不完善, 缺乏系统性和完整 性。

一方面, 我国现有的这方面法律法规比较零 散, 大多都是针对某个特定领域而制定的, 国家的 许多规章制度含有关于食品安全的相关内容, 但没 有形成一个有机的整体, 这就极易导致食品生产、 加工、销售、消费某个环节缺乏相应的法律法规规 范和调整, 而某些方面或环节, 不同的法律法规又 有重复规定, 这样在流通环节和农产品的再次加工 中给违法分子以可乘之机 ${ }^{[9]}$ 。

3.5.3 食品安全的技术支撑体系不完善。

由于我国还是发展中国家, 限于科技发展水

平, 相关食品安全检测技术、检测设备都还比较落

后, 同时我国食品的安全标准设定较低, 许多的标 准没有建立在科学的风险分析基础上, 不利于食品 安全监管的进行。

3.5.4 食品安全的各主体未充分发挥相应的责任。

首先, 企业的社会责任不高, 自律意识不强, 表现在生产、加工、销售过程中不能够严格地遵循 食品安全法律法规和标准, 为追求经济利益极易违 反行业道德和标准甚至相关的法律法规。其次, 广 大消费者食品安全意识比较淡薄, 相关知识缺乏 [10]。

\subsection{5 缺乏一套行之有效食品安全预警体系}

食品安全预警是指通过对食品安全隐患的监 测、追踪、量化分析、信息通报预报等，建立起一 整套针对食品安全问题的功能体系。对潜在的食品 安全问题及时发出警报, 从而达到早期预防和控制 食品安全事件、最大限度地降低损失、变事后处理 为事先预警的目的 ${ }^{[1]}$ 。

食品安全预警系统是食品安全控制体系不可缺 少的内容, 是实现食品安全控制管理的有效手段。

食品安全预警通过指标体系的运用来解析各种食品 安全状态、食品风险与突变现象, 揭示食品安全的 内在发展机制、成因背景、表现方式和预防控制措 施, 从而最大限度地减少灾害效应, 维护社会的可 持续发展。预警的关键在于及时发现高于预期的食
品安全风险, 通过提供警示信息来帮助人们提前采 取预防的应对策略 ${ }^{[12]}$ 。

目前我国还缺乏一套行之有效的食品安全预警 体系为消费者提供充足、可靠的安全信息; 及时发 布食品安全预警信息、帮助社会公众采取防范措 施; 对重大食品安全危机事件进行应急管理, 尽量 减少食源性疾病对消费者造成的危害与损失。

\section{4. 风险分析在现代化农业条件下发达国家的应用}

近年来, 发达国家为了提高食品安全监管效 率、保护消费者健康和促进食品与农产品国际贸 易, 在食品安全风险分析制度建设方面取得了很大 进展。食品法典委员会和一些发达国家开展了疯牛 病(BSE)、沙门氏菌、李斯特菌、O157: H7 、二恶 英、多氯联苯、丙烯酰氨等的系统研究 ${ }^{[13]}$, 已经形 成了化学危害物、微生物、真菌毒素等风险分析指 南和程序。当前风险评估技术已发展到能够对多种 危害物同时形成的复合效应进行评估，并且更加注 重随机暴露量的评估。另外, 国际社会对转基因食 品(GMO)的安全性评价问题也形成了评价原则和程 序，以美国、德国、日本等 3 种农业现代化道路国 家情况为例, 可看出风险分析在各发达国家的应用 情况和特点。

\section{1风险评估机构独立, 与风险管理职能分工明确。}

美国的食品安全风险分析由卫生部食品药品管 理局(FDA)、农业部(USDA)、环境保护署、海关与 边境保护局等按照各自的职能, 实行共同监管,

USDA 负责肉类和禽类等食品的管理, 监督食品安 全法规的执行。卫生部食品药品管理局(FDA)负责其 他类食品和进口食品的管理, 制订相关法规和最高 残留限量(MRLs)等食品安全标准。此种多机构联合 监管的模式体现在联邦政府、州和地方等层面, 可 开展纵向的风险分析, 也可跨机构进行横向合作。 早在 1997 年 5 月, 美国就成立了风险评估联盟, 旨 在协调风险评估, 交流信息, 促进食品特别是动物 源食品的风险评估和科学研究。

2002 年 11 月, 依据《消费者健康保护和食品 安全重组法案》, 德国联邦消费者保护、食品与农 业部设立了联邦风险评估研究所和联邦消费者保护 与食品安全局。其中, 联邦风险评估研究所负责风 险评估和风险交流；联邦消费者保护与食品安全局 负责风险管理及与联邦、其他国家及欧盟机构的协 调。联邦风险评估研究所下设 7 个部门, 每个部门 下设工作组或实验中心，负责本领域内风险评估的 相关工作, 通过各部门的明确分工与相互协作, 联 
邦风险评估研究所可完成对食品中生物与化学的风 险评估, 并以风险评估结果为基础提出降低风险的 管理选项, 为联邦政府部门和其他风险管理机构提 供建议。

2003 年 7 月 1 日, 依据《食品安全基本法》, 日本成立了食品安全委员会, 专门从事食品安全风 险评估和风险交流工作。该委员会由 7 名食品安全 专家组成, 委员全部为民间专家, 经国会批准, 由 首相任命, 任期 3 年。委员会下设事务局和专门调 查会, 事务局负责日常工作, 专门调查会负责专项 案件的检查评估。食品安全委员会的主要职责是实 施食品安全风险评估; 对风险管理部门进行政策指 导与监督; 负责风险信息的沟通与公开。从发达国 家的经验来看, 独立的风险评估确保了食品安全立 法与标准制定的科学性, 为有效的风险管理提供了 依据 ${ }^{[14,15]}$ 。

\section{2 风险管理较为有效}

在管理思想上，各发达国家一改过去基于事后 处置的管理体系，转为进行事前干预的有效管理。 另外从政策效率角度出发, 让生产者承担生产安全 食品的主要责任, 让政府实行有效的监督, 为政府 的管理节约了大量的人力、物力与财力。

在管理体制上, 国际食品安全管理体制的核心 是加强食品安全管理各部门之间的协调。为此大多 数国家改变了原先按食品品种或按生产阶段来划分 监管部门的职能的分割式体制, 将原有的食品安全 管理部门统一到一个独立的食品安全机构, 如加拿 大、德国、丹麦和澳大利亚等国家。由于分割式体 制转变为统一式体制的运作成本较高, 因此, 也有 许多国家食品安全管理机构依然分布在不同部门, 但通过明确分工来协调实现食品安全, 比如美国和 日本。

在管理手段上, 发达国家更多的是鼓励企业进 行自我质量管理。食品加工企业进行自我质量管理 的主要手段有良好生产规范（GMP）、良好卫生规 范(GHP)、危害分析与关键控制点(HACCP)。其中, GMP 和 GHP 是 HACCP 实施的前提条件, 而 HACCP 则融合了风险评估和风险管理两个阶段, 既 是企业控制食品安全的方法, 又是政府进行管理的 有效手段, 为发达国家广泛采用。美国是最早应用 HACCP 系统的国家, 目前美国海产品的加工者、肉 禽产品的加工者及果蔬汁加工者都被要求执行 HACCP 系统, 同时欧共体、日本、澳大利亚、新西 兰也将实施 HACCP 体系作为解决本国食品安全问 题的有效措施 ${ }^{[16]}$ 。

\section{3风险交流比较充分}

食品安全管理的最终目标是减少食源性疾病的 发生, 保护公众健康, 其中消费者作为食品安全管 理的最终服务目标, 对政策的理解影响着他们对市 场的信心，消费者在整个风险分析中占据着十分重 要的地位, 因此也受到了各国政府的重视。

在德国, 风险交流作为联邦风险评估研究所的 一项法定任务, 应向消费者提供有关食品和产品中 可能存在及已被评估的风险信息。当需要在较大范 围内通知公众时, 除媒体外, 消费者建议中心、产 品比较团体、消费者保护、食品与农业信息服务部 都会成为风险交流的重要手段。为了实现风险信息 交流的持续和互动, 联邦风险评估研究所定期组织 专家听证、科学会议及消费者讨论会, 并面向一般 公众、科学家和其他相关团体公开其评估工作与结 果, 并通常会在其网站上公布专家意见和评估结 果, 还积极寻求以简易的方式与普通公众对评估过 程进行交流, 并向消费者提供可见和可用的科学研 究成果。通过全面的风险交流, 一方面, 尽早发现 潜在的健康风险并及时通知有关部门和消费者; 另 一方面, 参与交流的各相关方会对风险评估的过程 与结果进行讨论, 通过工作的透明度, 在风险评估 涉及的各方之间建立起足够的信任。在日本人，食 品安全委员会主要通过召开国际会议与国外政府、 国际组织和相关部门进行风险交流, 来交换各方意 见和建议, 确定自身食品安全风险评估的方向。同 时通过网站、热线和专人信息采集与公众进行风险 交流, 来听取消费者和公众的意见与建议。食品安 全委员会通过每周 1 次公开召开委员会议, 并在其 网站公布会议议程来保证实施风险评估的透明性。 通过建立 “食品安全热线”, 专门用来接收日本民 众对于食品安全的要求和意见。同时, 食品安全委 员会从各县选拔任命了 470 名 “食品安全监督 员” , 监督员通过发放调查问卷来了解食品安全事 件引起人们关注的程度, 及时汇报相关信息, 并且 协助各地方组织进行信息交流 ${ }^{[15]}$ 。

\section{5. 风险分析在传统农业条件下的应用情况}

中国的食品安全风险分析主要参考美国模式。 我国在传统农业条件下的食品安全管理方面最初应 用风险分析是在 20 世纪 90 年代中后期, 在农产品 质量安全方面谈论风险分析最初始于 2001 年。2002 年农业部也专门成立了农业转基因生物安全评价专 家委员会, 对农业转基因植物、动物和微生物开展 风险评估和安全评价。2006 年 11 月 1 日施行的 《农产品质量安全法》确立了风险评估的法律地 
位, 要求把风险评估结果作为制定农产品质量安全 标准的重要依据, 农业部门要根据风险评估结果采 取相应的管理措施。2009 年施行的《中华人民共和 国食品安全法》也对食品安全风险评估及其结果的 利用做出明确规定, 表明我国的风险评估已进入法 制轨道。目前, 正在修订的《食品卫生法》将首次 增加食品安全风险评估这一重要内容, 政府有关监 管部门可以根据风险评估的结果对高风险的食品重 点监管。新修订的《食品中农药最大残留限量标 准》(GB2763-20 05) 和《食品中污染物限量》(GB 2762-2005)较大程度地引用了 $\mathrm{CAC}$ 标准的风险评估 数据, 等同性均提高了 $85 \%$ 以上。兽药典也大幅度 采用 $\mathrm{CAC}$ 标准的风险评估数据, 等同性均提高到了 $41.4 \%^{[17]}$ 。 《农业转基因安全评价管理办法》规定对 人类、动植物、微生物和生态环境构成的危害进行 风险评估和分析。《兽药注册办法》规定: 农业部对 申请进口注册的兽药进行风险分析, 经风险分析存 在安全风险的, 不予注册。

近年来，我国商务、卫生、农业和检验检疫部 门针对食品方面的危害分析做了大量工作，检验检 疫部门结合我国进出口贸易中出现的热点问题和国 际热点问题在口岸开展了应用实践, 如对酱油中三 氯丙醇; 苹果汁中甲胺磷、乙酰甲胺磷残留; 禽 肉、水产品中氯霉素残留; 冷冻加工水产品中金黄 色葡萄球菌及其肠毒素; 油炸马铃薯食品中丙烯酰 胺; 水产品中金属异物; 牡蚛食用中感染副溶血性 弧菌; 进境冻大马哈鱼携带溶藻弧菌可能影响人体 安全和水产动物健康的风险评估等等的风险评估 [18]。

然而, 由于起步较晚, 法规不健全, 风险分析 缺乏完整、配套的制度保障。食品安全风险分析只 停留在对某一种食品和产业链的某一环节上, 风险 分析体系尚未形成。中央一级的相关工作由农业 部、卫生部、国家质量监督检验检疫局等多部门负 责, 没有设立专门的机构, 风险评估能力弱。信息 传播机制不完善，风险交流不充分。食品行业缺乏 诚信, 影响风险分析效果。

5.1 传统农业条件下食品安全风险分析工作相关的法 律法规不完善

由于我国在传统农业条件下应用食品安全风险 分析的起步时间较晚, 又多以国外的相关研究为基 础, 在我国尚未摸索出一套适应我国国情的, 能够 有效指导传统农业条件下进行食品安全风险分析工 作的法律法规。
5.2 传统农业条件下的食品安全风险分析框架尚未形 成

我国现在并不缺食品安全风险分析的专业人 员, 缺的是一种制度保障。比如, 风险评估需要大 量的监测数据, 而我国并没有完善的、畅通的监测 网络, 无法有效地变事后监管为事前预防。在以往 的食品安全事件的处理上, 往往只是停留在对某一 种食品或者一起食品安全事件的评估上, 没有专门 的权威机构从整体上对该类食品的整条生产消费链 的风险进行评估。这种方式一味的追求当前利益和 效果，治标不治本无异于 “饮扸止渴”。

5.3 传统农业条件下适应食品安全风险分析的外部环 境尚未成熟

在我国农业区域辽阔、各地农业资源差异巨 大、农业技术应用不充分、农业科技应用水平参差 不齐、农村劳动力数量庞大而素质低下和农业投入 不足等客观现实下，虽然有时用了风险评估的方 法, 但由于样本量小, 检测监测手段有限, 食品安 全风险分析体系所需的信息交流渠道闭塞，技术人 员缺乏, 使得获得的相关数据品质不高, 这些因素 对于评价水平、评价结果有很大的负面影响。

5.4 传统农业条件下的食品行业缺乏诚信, 影响风险 分析效果

开展食品安全风险分析必须有一个诚实信用的 环境。技术、设备、人才、资金等是食品安全风险 分析的重要硬件, 而企业诚信是食品安全分析的首 要软件。如果食品生产者为了追求经济利益, 不顾 消费者健康, 向食品中添加有毒有害物质, 那么再 完美的食品安全风险分析也无法保障食品安全。食 品安全风险分析在发达国家得到很好应用, 一个重 要的原因是发达国家食品行业的诚信水平普遍较 高, 保证了食品安全法律标准严格的执行 ${ }^{[4]}$ 。

\section{6. 传统农业条件下进行食品安全风险分析的建议}

6.1 借鉴发达国家应用风险分析的先进经验, 构建适 合我国国情的食品安全风险分析体系.

我国作为 WTO 成员, 应遵循国际组织相关规 定, 积极进行国际交流, 及时了解并学习发达国家 的先进经验, 逐步建立、完善适合我国国情的食品 安全风险分析体系。

6.2 建立和完善食品安全相关的法律法规体系 
国家立法部门要根据现阶段我国食品安全问题 的实际情况, 对现有的食品安全法律、法规、条 例、标准、规范等进行整合, 使之系统化; 同时需 要制定、出台相应的新的食品安全法律法规, 拒绝 法律的空白或薄弱环节和领域, 使之完整化。在食 品安全监管过程中, 一方面用法律形式明确各级相 关政府部门的职责, 针对具体问题要确保法律的可 操作性, 做到为实际问题服务; 另一方面用法律形 式明确食品领域生产者、消费者等主体的权利义务 关系。从而真正做到食品安全有法可依, 有法必 依。

6.3政府、企业、消费者协调统一发挥各自作用

政府应致力于食品安全法规的建立和实施, 在 法律的框架范围内改进国家食品安全管理机构的组 织形式和管理模式。同时协调与国际组织, 尤其是 国内涉及食品安全的各行政机构的信息收集、交换 和整合工作。收集国内外的有关信息, 尽早掌握事 态的发展，协调有关行政机关迅速应对。

食品企业应该在生产过程中充分发挥 HACCP 管理的作用。HACCP 通过对整个食品链, 包括原辅 材料的生产、食品加工、流通、乃至消费的每一环 节中的物理性、化学性和生物性危害进行分析、控 制以及控制效果验证的完整系统。风险分析是对 HACCP 管理体系的进一步补充和完善, 也是实行 HACCP 管理体系的基础。作为主体的企业的自律行 为和实践经验, 行业协会的监督约束, 均为进行风 险管理提供了宝贵的经验。

消费者对风险分析的认知程度是实施风险分析 的基础。随着社会发展和人们对食品安全的了解程 度及关注程度的不断提高, 借助行业协会和团体会 议的交流、网页、公开发行的刊物等媒体的作用, 通过消费者的积极参与和信息交流, 最终能为实施 风险分析奠定良好的基础。[19]

6.4改善食品安全监督管理模式, 加大食品安全的技 术支持

在相关法律法规的调整下, 执法部门要把法律 法规落实到位。一是要整合执法力量, 形成统一、 权威、高效的食品安全监管模式, 解决多头执法、 重复执法等问题。二是要加快食品安全标准体系建 设。政府有关部门应加快健全食品安全标准体系, 加快检验检疫农药残留限量、食品添加剂等方面的 食品质量安全卫生标准的制定修订步伐。包括与国 际标准、国家标准以及行业标准相配套的地方标准 体系建设, 并向农民、企业推荐和介绍国际标准和 国外先进标准, 积极引导和鼓励他们达到先进标
准。三是要努力提高食品安全检测、控制技术，完 善检测网络体系。一方面加大投入, 提高我国食品 特别是农产品安全生产和检测技术, 强化农药残留 检验手段和技术; 另一方面加大投入，努力更新完 善检验检测设备, 同时管理部门可以把农业教学、 农技推广等部门的检验设施充分利用起来，从而形 成完整的检测网络。

\section{5 实施风险评估和风险管理职能分离机制}

风险评估不仅是风险分析的核心，也是进行风 险管理的科学依据。风险评估这一个科学过程, 应 由科学家独立完成, 不应受政治、经济、文化的影 响。而风险管理是由政府完成的一个决策过程, 必 然会受政治、经济、文化的影响。所以为保障风险 评估的科学性、客观性、透明度和有效性, 应将风 险评估和风险管理职能分开, 成立专门的风险评估 机构, 遵循内外一致的原则进行风险评估 ${ }^{[20]}$ 。

6.6 建立科学、统一的食品安全检测体系

食品安全检验检测是食品安全风险管理的重要 手段之一, 它为食品安全风险管理提供重要的技术 支持和管理政策依据。目前, 我国食品安全检验检 测体系的基础框架虽然已经初步形成, 但是食品安 全检验检测机制、设施、技术力量和手段等还不够 完善。当前存在的突出问题是质监、卫生、农业等 部门往往按照本部门颁布的有关规定进行检测, 检 测的结果比较独立, 部门与部门之间缺乏良好的共 享和互认机制。我国必须建立科学、统一的食品安 全检测体系, 强化技术监管, 加强食品安全风险管 理。通过监测收集一批与中国农产品质量安全相关 的分析数据, 根据中国农产品质量安全预警体系, 适时公告一批高风险危害因子和对应的产品, 提请 公众关注和警觉, 政府部门也要采取相应的防范措 施；以正确引导生产消费，增强消费信心 ${ }^{[17]}$ 。

\section{7. 结论}

一般来说, 传统农业下的生产模式与食品安全 问题并没有直接的关系, 因为即便在生产力十分落 后的封建社会, 人们对大部分直接种植养殖出的食 用农产品的 “安全” 问题也基本不用担心。然而随 着经济的发展, 城市化的推进, 传统农业生产条件 下引发的食品安全问题却越来越引起人们的关注, 这期间除了农业种植养殖自身发生了一些改变之外

(如越来越多地使用农药、化肥和各类饲料添加 剂; 以及现代市场经济对传统农业生产条件下的道 德、诚信规范的冲击），而从食品安全风险评估的 
角度来看, 传统农业生产与现代物流、加工业的信 息化管理严重脱节, 是引发食品安全诸多问题的重 要原因之一。这是因为引发食品安全的因素众多, 对其的监管是一个十分复杂的系统工程, 问题的产 生即与源头的生态环境保护程度和农产品种植养殖 的过程有关, 也与运输、加工方式, 以及消费者对 食品安全的认知程度紧密相连。进行食品安全风险 评估首先需要有效地获得食品生产、食品供应、食 品物流与食品需求等四个主要领域产生的检验检 测、问卷调查等相关数据。由于我国广大农村目前 信息化管理水平低下, 有些地方在这方面基本就是 空白, 因此很难保证风险评估的基本数据的获取; 加上经济飞速发展下的利益诱惑, 诚信体制严重缺 失, 更给食品安全监管过程中的源头控制工作带来 了巨大的困难。

\section{参考文献}

[1] 石兴, 自然灾害风险可保性理论及其应用研究, 北京师 范大学博士学位论文, 2009。

S. Xing, Insurability Study and its Application on Natural Disasters, PhD thesis, Beijing Normal University, 2009.

[2] 张兆本, 公共经济学, 北京: 人民出版社, 2005

Z. B. Zh ang, Public E conomics, Beijing: The People's Press, 2005

[3] 曾莉娜, 中国食品安全风险分析机制研究, 上海师范大 学, 2010

[4] 吴培, 许喜林, 张毅, 食品安全风险分析与应用, 现代 食品科技，2006，22(04)，200-203

[5] 魏益民, 基于风险分析原理的中国食品安全法规体系建 设，中国食物与营养，2009，8:11-13

[6] 徐成德, 我国开展食品安全风险分析的问题与对策, 农 产品加工，2009，2:61-66

[7] 袁宗辉, 我国应重视食品安全风险分析, 华中农业大学 学报, 2010, (3):8-12

[8] Ariane Koing, Harry A. Kuiperc , Hans J. P. Marvin, et al.The safe food s framework for improved risk analysis of foods. Food Control 2010， 21:1566-1587

[9] http://www.gzu521.com/paper/article/industry/200607/2272 7.htm

[10] 张卫斌, 顾振宇, 基于食品供应链管理的食品安全问题 发生机理分析，食品工业科技，2007，28(1):215-220

[11] 张森富, 赵婷, 现阶段我国食品安全问题的成因及其对 策研究, 长春理工大学学报, 2010, 23(1): 38-39

[12] R.B.Tompkin. Interactions be tween gov ernment and industryfood safety activities. Food Control, 2001, 12:203-207

[13] 叶存杰, 基于.NET 的食品安全预警系统研究, 科学技 术与工程, 2007, (2):258-260

[14] 杨君, 黄丽, 袁利鹏, 关于完善我国食品安全风险预警 系统的思考, 食品科技, 2009 年第 34 卷第 12 期

[15] 刘春霞, 浅谈食品安全风险分析, 2008 年度标准化学 术研究论文集, 2009
[16] 滕月, 发达国家食品安全规制风险分析及对我国的启 示，哈尔滨商业大学学报，2008，(5):55-57

[17] 王芳, 陈松, 钱永忠, 国外食品安全风险分析制度建立 及特点分析，世界农业，2008，(9); 44-47

[18] 周应恒，彭晓佳，风险分析体系在各国食品安全管理中 的应用，世界农业，2005(3): 4-6

[19] 范梅华, 张建华, 风险分析-我国食品安全管理的必由 之路，中国禽业导刊，2007，24（24）

[20] 刘志英, 风险分析一我国食品安全管理新趋向, 内蒙古 科技与经济，2006，4(5): 141- 143

[21] 孙俐, 贾伟, 食品安全风险分析的发展与应用, 食品研 究与开发，2008，29（6）

[22] 王成, 陈小莉, 食品安全风险分析是我国食品安全管理 必经之路, 上海食品药品监管情报研究，2008，12(95) 